\title{
Scanning x-ray excited optical luminescence microscopy in GaN
}

\author{
G. Martínez-Criado ${ }^{a)}$ \\ European Synchrotron Radiation Facility, 38043 Grenoble, France \\ B. Alen \\ Microelectronics Institute Madrid, CNM-CSIC, 28760 Tres Cantos, Spain
}

A. Homs, A. Somogyi, ${ }^{\text {b) }}$ C. Miskys, ${ }^{\text {c) }}$ and J. Susini

European Synchrotron Radiation Facility, 38043 Grenoble, France

J. Pereira-Lachataignerais

Chemical and Environmental Research Institute, CSIC, 08034 Barcelona, Spain

J. Martínez-Pastor

Materials Science Institute, University of Valencia, 46100 Burjasot, Spain

(Received 21 September 2006; accepted 20 October 2006; published online 29 November 2006)

In this work, an imaging tool to investigate optical inhomogeneities with site and chemical sensitivities has been integrated in a hard x-ray microprobe. Freestanding GaN and epitaxially grown GaN:Mn on $\alpha-\mathrm{Al}_{2} \mathrm{O}_{3}$ are used to exploit the unprecedented scanning $\mathrm{x}$-ray excited luminescence technique. Optical images of the radiative recombination channels are reported for several impurities and defect centers in sapphire and GaN compounds. Within the experimental accuracy, a visible nonuniformity characterizes the Mn centers in good correlation with former x-ray fluorescence map. Expanding the microprobe versatility, x-ray absorption spectroscopy in both photon collection modes (x-ray excited luminescence and $\mathrm{x}$-ray fluorescence) is finally presented from a freestanding GaN layer. (C) 2006 American Institute of Physics. [DOI: 10.1063/1.2399363]

The enormous demand for superior optoelectronic devices has systematically stimulated the application of further optical characterization techniques. Combining the cuttingedge lasers, advanced detectors, and other high technology components, we are able today to assemble a spectroscopic instrument with characteristics that were hardly achievable a decade ago. In the present work, we propose a photoluminescence (PL) approach based on x-ray core-level excitation using a hard x-ray microbeam. As an excitation light source, synchrotron-based microprobes offer unique benefits. Its brightness can approach 1000 times that of thermal sources. Moreover, its electromagnetic spectrum usually spans a wide wavelength range, from a few $\mathrm{keV}$ up to several tens of $\mathrm{keV}$. Thus, by carefully selecting the incident energy, the PL signal can be enhanced and buried layers can be investigated within the device heterostructures. Therefore, several potential experiments could benefit in an exceptional way by this scanning optical probe. X-ray excited optical luminescence (XEOL) imaging of carrier traps and/or optical active centers, nonlinear luminescence spectroscopy on the micrometer scale, and polarization-dependent studies of optical processes are just a few examples. So far, a typical hard x-ray microscope provides significant information in terms of elemental specificity [X-ray fluorescence (XRF)], ${ }^{1}$ chemical mapping, ${ }^{2}$ coordination chemistry [x-ray absorption near-edge structure, (XANES)], and site symmetry (x-ray diffraction and angular dependent XANES). ${ }^{4}$ If a PL spectrum is also recorded at each point, optical maps may be produced simultaneously that show complementary properties spatially resolved. The effect of doping, strain, and disorder can be studied with high sensitivity by PL. ${ }^{5}$ For crystal quality evaluation, it is also a

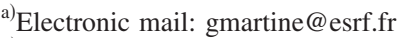

${ }^{b)}$ Present address: Synchrotron Soleil, 91192 Gif-sur-Yvette, France.

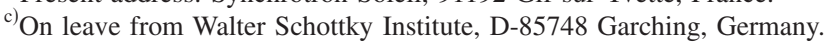

straightforward tool for quantitative estimation of residual stress, residual chemical species in alloys, identification of minority phases, etc.

Therefore, addressing the need for a multipurpose tool that equips a hard x-ray microanalysis end station with a luminescence detection probe (Fig. 1), here we introduce such an optical-sensitive scheme at the beamline ID22 of the European Synchrotron Radiation Facility. ${ }^{1}$ The experimental layout of the scanning PL spectroscopy at ID22 microprobe is shown in Fig. 1. In order to study the radiative recombination channels in highly demanded wide band gap materials, UV-visible wavelength detection range was prioritized. Thus, to bring the UV-visible light into an optical spectrometer (Ocean Optics, Inc.), aspheric collection optics and fiber optic accessories are incorporated. Located at the control cabin, which adds flexibility to the experimental design, the monochromator separates the luminescence into its constituent wavelengths onto a linear charge-coupled device (CCD) $\mathrm{Si}$ detector. Finally, the spectrometer controller and data acquisition software makes optimum use of the collected pho-

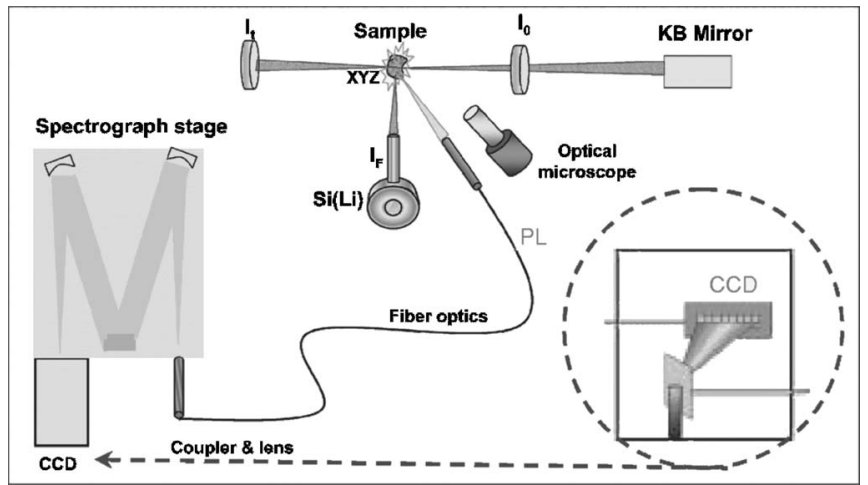

FIG. 1. Schematic layout of the scanning x-ray excited optical luminescence arrangement. 


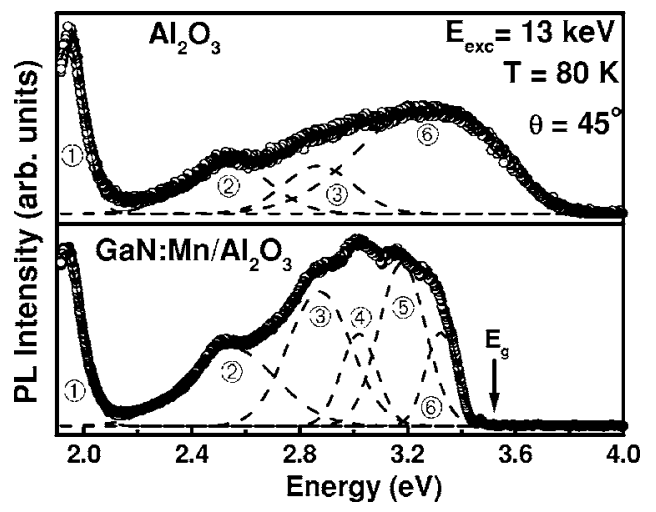

FIG. 2. X-ray excited luminescence spectra taken at $13 \mathrm{keV}$. Upper part: Luminescence of the $\mathrm{Al}_{2} \mathrm{O}_{3}$ backside excited. Lower part: Luminescence of both $\mathrm{GaN}: \mathrm{Mn}$ and $\mathrm{Al}_{2} \mathrm{O}_{3}$ overlapped, nitride film side excited.

tons. To prevent thermal ionization of the optically active centers and minimize the broadening of sharp spectral features by lattice vibrations, an Oxford LN2 cryostream is also used.

A $1 \mu \mathrm{m} \mathrm{GaN:Mn} \mathrm{layer}([\mathrm{Mn}] \approx 11 \%)$ grown on $\alpha$ $\mathrm{Al}_{2} \mathrm{O}_{3}(\sim 500 \mu \mathrm{m}$ thick) was chosen for this study because of the great potential of both materials for advanced electronic devices. In addition, a precise knowledge of the material properties is a prerequisite for understanding the physical phenomena involved in the XEOL. By hard x-ray microanalysis, the former experiments clearly revealed the formation of Mn clusters on the micrometer scale. ${ }^{2}$ The strong polarization-dependent XANES features also showed the preservation of the hexagonal symmetry in both cluster-free and Mn-rich regions, ${ }^{4}$ while donor- and acceptor-bound exciton lines dominated the laser excited luminescence at $6 \mathrm{~K}^{3}$ Here, as a continuation of the previous reports, ${ }^{2-4}$ its optical homogeneity will be also examined by XEOL mapping. The average $x$-ray excited luminescence spectra taken at $13 \mathrm{keV}$ over a $100 \times 65 \mu \mathrm{m}^{2}$ scanned area are shown in Fig. 2 . In the upper part, the background luminescence excited from the $\mathrm{Al}_{2} \mathrm{O}_{3}$ backside is shown. Its spectral decomposition shows four bands located at $1.9 \mathrm{eV}(1), 2.6 \mathrm{eV},(2), 2.9 \mathrm{eV}$ (3), and $3.3 \mathrm{eV}(6)$. The last three emissions are associated with residual $\mathrm{Ti}$ impurity and point defects in $\mathrm{Al}_{2} \mathrm{O}_{3}$, ${ }^{6,7}$ while the narrow band at $1.9 \mathrm{eV}$ is tentatively attributed to the unresolved transition of the trace impurity $\mathrm{Fe}^{3+}:{ }^{4} T_{1} \rightarrow{ }^{6} A_{1} \cdot{ }^{8}$ On the lower part, the sample has been reversed to excite and collect through the GaN side. The spectrum shows the absorption of the high energy tail of the luminescence from the sapphire $\left(E_{g}=9 \mathrm{eV}\right)$ by the $\mathrm{GaN}\left(E_{g}=3.42 \mathrm{eV}\right)$. The spectral analysis suggests the presence of six peaks, where both $\mathrm{Al}_{2} \mathrm{O}_{3^{-}}$and $\mathrm{GaN}: \mathrm{Mn}$-related transitions are overlapped. In recent studies on GaMnN with low Mn content, the PL band around $3.0 \mathrm{eV}$ (4) accompanied with another transition at $2.5 \mathrm{eV}$ (2) has been assigned to Mn-related recombinations. ${ }^{9,10}$ Although the typical yellow band associated with intrinsic defects $\left(V_{\mathrm{Ga}}\right)$ is a common competitive GaN recombination channel, ${ }^{11}$ the average spectrum in Fig. 2 does not exhibit any transition at $2.2 \mathrm{eV}$. However, the typical donor-acceptor pair recombinations, attributed to oxygenrelated complexes and vacancy defects, are superimposed at $2.8 \mathrm{eV}$ (3) and $3.2 \mathrm{eV}$ (5) respectively. ${ }^{12,13}$

To study the two-dimensional spatial distribution of specific recombination channels, optical maps are obtained on a pixel-by-pixel basis by a multiple-Gaussian fitting procedure Downloaded 08 Oct 2009 to 161.111.235.123. Redistribution subject

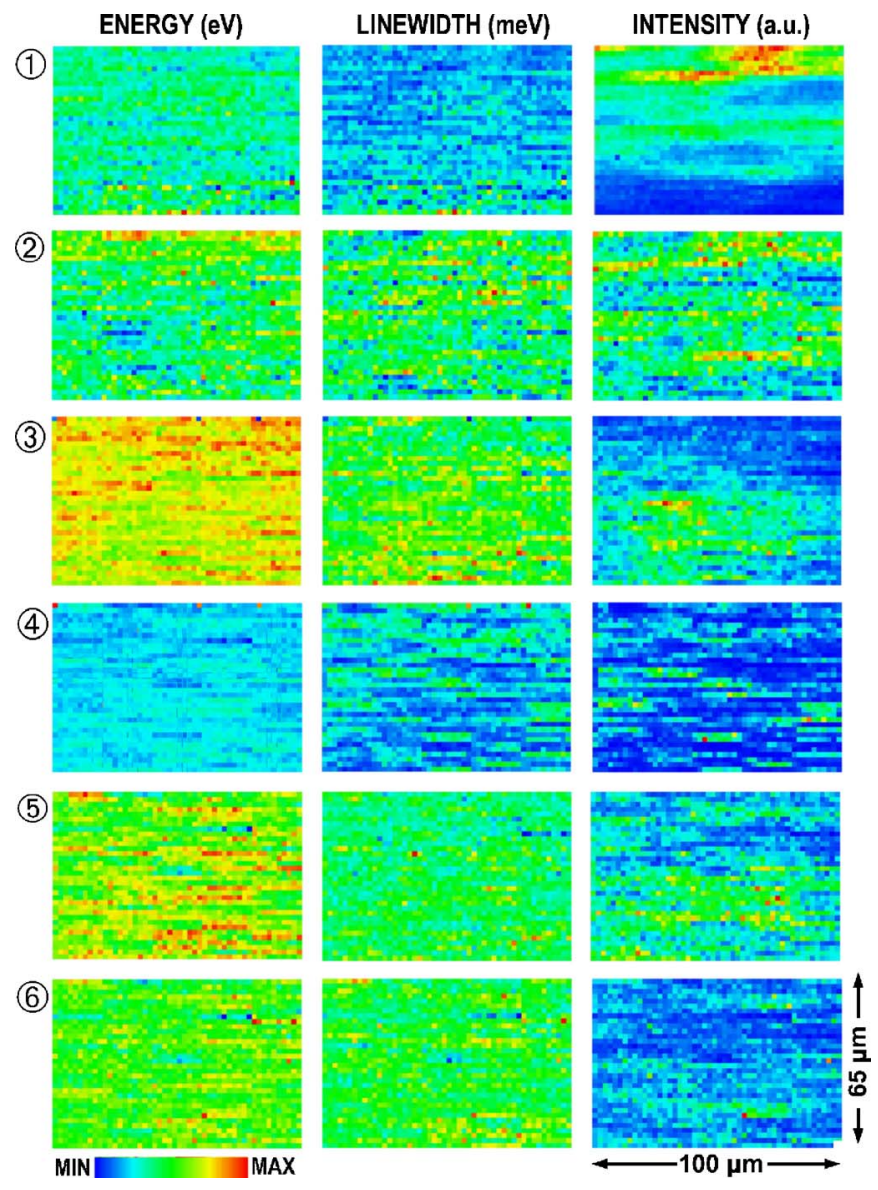

FIG. 3. Spatial variation of the different transition energies, linewidths, and integrated intensities (defined in Fig. 2) recorded over a $100 \times 65 \mu \mathrm{m}^{2}$ scanned area of $\mathrm{GaN}: \mathrm{Mn} / \mathrm{Al}_{2} \mathrm{O}_{3}$. The values of the different color scales are listed in Table I in the graph, blue represents low values, red high values).

applied to each PL spectrum. The resulting spatial variations of the different transition energies, linewidths and integrated intensities recorded over the $100 \times 65 \mu \mathrm{m}^{2}$ are displayed in Fig. 3. The mean average for each magnitude and its relative standard deviation are listed in Table I. Figure 3 illustrates optical features that are not visible through conventional PL but becomes apparent from XEOL spectral analysis. In general, the integrated intensity maps show that nonuniform luminescence patterns characterize the electron-hole recombination over the sample. Since the noise in our current spectroscopic system is not negligible, different improvement steps are presented below. From the highest intense map (1), a strong spatial variation of density of $\mathrm{Fe}^{3+}$ ions and their corresponding radiative transition rates is observed.

TABLE I. Mean average and relative standard deviation (in \%) of the energy (in eV), linewidth (in $\mathrm{meV}$ ), and integrated intensity (in a.u.) of the different PL transitions on the color maps.

\begin{tabular}{cccccccc}
\hline \hline & \multicolumn{3}{c}{ Energy linewidth } & & \multicolumn{2}{c}{ Intensity } \\
\cline { 2 - 3 } Peak & $(\mathrm{eV})$ & $(\%)$ & $(\mathrm{meV})$ & $(\%)$ & & $($ a.u. $)$ & $(\%)$ \\
\hline 1 & 1.93 & 0.52 & 40.20 & 16.67 & & 5577 & 54.29 \\
2 & 2.59 & 1.93 & 70.45 & 25.20 & & 4001 & 39.57 \\
3 & 2.95 & 3.72 & 32.60 & 31.90 & & 3601 & 64.73 \\
4 & 3.01 & 3.81 & 21.80 & 51.83 & & 2216 & 80.82 \\
5 & 3.22 & 1.71 & 16.30 & 39.26 & & 1896 & 72.05 \\
6 & 3.32 & 0.90 & 10.35 & 33.34 & & 977 & 83.21 \\
\hline \hline
\end{tabular}




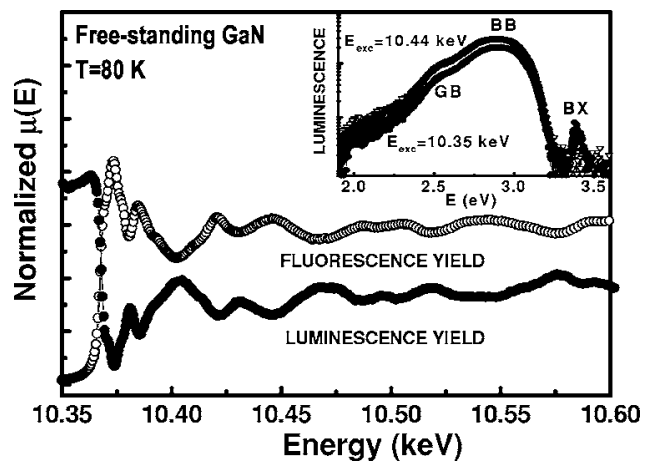

FIG. 4. Normalized $x$-ray absorption spectra near the Ga $K$ edge taken in both $\mathrm{x}$-ray excited optical luminescence and $\mathrm{x}$-ray fluorescence modes from a freestanding GaN layer. The inset shows the PL spectra excited with x-ray energies below and above the Ga $K$ edge.

Within the detection limit of our system, the integrated intensity map (4) exhibits an inhomogeneous projection of Mn optical centers in agreement with the Mn incorporation revealed by $\mathrm{XRF}^{2}$. The linewidth maps, on the other hand, display the impurity induced broadening mechanisms and their effects on the emission line shapes. Given the experimental accuracy, mostly randomly distributed uniform layers illustrate the transition linewidths. But despite probing different sample depths, image (4) also shows a more-or-less consistent result with the XRF map. ${ }^{3}$ Except the emission wavelength for $\mathrm{Fe}^{3+}$, most of the energy-based maps suggest a small but apparent residual strain variation over the sample (see Table I). The long range Coulomb interaction has a minor influence on the intra-atomic transition that remains almost constant along the map.

To avoid the overlapping of both GaN- and substraterelated luminescence, in Fig. 4 the Ga $K$ edge XANES in fluorescence and $\mathrm{x}$-ray excited luminescence detection modes have been plotted for a freestanding GaN layer. ${ }^{14}$ The inset displays the PL spectra of the metal-organic chemicalvapor deposition grown $\mathrm{GaN}$ film excited with $\mathrm{x}$-ray energies above and below the Ga $K$-edge. The spectra consist of two broad bands with insensitive shapes to the excitation energy. At $2.8 \mathrm{eV}$ the blue emission band (BB) dominates the luminescence, whereas at $2.5 \mathrm{eV}$ the green band (GB) commonly attributed to defects can be observed as a shoulder. ${ }^{11}$ The bound exciton (BX) lines appear at higher energies with very low intensity, representing intrinsically the real sample temperature. Both self-absorption corrected spectra were recorded at an incident angle of $\theta=45 \pm 5^{\circ}$ with respect to the sample surface. The BB and GB bands follow the same tendency with the x-ray energy. As expected for a thick and totally absorbing sample (100 $\mu \mathrm{m}$ thick) under the used experimental geometry, in comparison with the fluorescence yield, the luminescence shows a negative edge jump with inverted extanded x-ray absorption fine structure oscillations. In accordance with the phenomenological theory proposed by Emura et al. ${ }^{15}$ and Gauthier et al. ${ }^{16}$ the negative edge jump suggests that the high-energy electrons excited from outer core states by x-rays at $\mathrm{Ga} K$ edge region contribute more to the optical luminescence than those from inner core states. In the anticorrelation behavior, similar features characterize both data except a slight difference in their relative peak intensities. Since the escape depth of the luminescence is smaller than the information depth of the fluorescence, Fig. 4 indicates that the Ga $K$ shell excitation does not create Downloaded 08 Oct 2009 to 161.111.235.123. Redistribution subject additional optical luminescence channels in the sample.

From both mapping and spectroscopic findings, in comparison with temperature dependent PL measurements, ${ }^{3,14}$ the results show that our system also suffers from a poor low-temperature effect. Sharper wavelength discriminations, which significantly improve the quantum efficiency of the different luminescence processes, are only achieved at much lower temperatures. A dedicated He microcryostat is necessary to minimize thermal disorder effects, guaranteeing a well-controlled low temperature environment. At the same time, a CCD detector with better noise performance, lower dark current (reached by cooling), and higher quantum efficiency is foreseen to obtain satisfactory signal-to-noise ratios.

In summary, the developed instrument for performing scanning luminescence spectroscopy with $\mathrm{x}$-ray microbeam was applied to $\mathrm{GaN}$. Despite the improvement steps of the technical setup as well as the energy relaxation and transfer processes involved in XEOL, relevant optical properties have been examined spatially and spectrally dependent in $\mathrm{Mn}$ doped $\mathrm{GaN}$ on $\alpha-\mathrm{Al}_{2} \mathrm{O}_{3}$. The PL scanning probe illustrated the nonuniform distribution of the $\mathrm{Fe}^{3+}$ ions and defect-related centers in sapphire, while a good agreement was obtained between the PL mapping experiment and formerly reported Mn pattern in GaN by XRF. Furthermore, the luminescence from a freestanding GaN film was monitored around the Ga $K$ edge, demonstrating that no additional radiative channels are created above the Ga $K$ shell.

Special thanks are due to Martin Hermann, Martin Eickhoff, and Martin Stutzmann (Walter Schottky Institute) for providing the sample used in this study. The authors are also grateful to Remi Tucoulou as well as Sylvain Laboure for their useful help.

${ }^{1}$ A. Somogyi, R. Tocoulou, G. Martínez-Criado, A. Homs, J. Cauzid, P. Bleuet, and A. Simionovici, J. Synchrotron Radiat. 12, 208 (2005).

${ }^{2}$ G. Martínez-Criado, A. Somogyi, A. Homs, R. Tucoulou, and J. Susini, Appl. Phys. Lett. 87, 061913 (2005).

${ }^{3}$ G. Martínez-Criado, A. Somogyi, M. Hermann, M. Eickhoff, and M. Stutzmann, Jpn. J. Appl. Phys., Part 2 43, L695 (2004).

${ }^{4}$ G. Martínez-Criado, A. Somogyi, S. Ramos, J. Campo, R. Tucoulou, M. Salome, J. Susini, M. Hermann, M. Eickhoff, and M. Stutzmann, Appl. Phys. Lett. 86, 131927 (2005).

${ }^{5}$ Handbook of Luminescence, Display Materials and Devices (American Scientific, Stevenson Ranch, CA, 2003), Vol. 1-3, p. 46.

${ }^{6}$ V. B. Mikhailik, H. Kraus, D. Wahl, and M. S. Mykhaylyk, Appl. Phys. Lett. 86, 101909 (2005).

${ }^{7}$ B. D. Evans, G. J. Pogatshnik, and Y. Chen, Nucl. Instrum. Methods Phys. Res. B 91, 258 (1994).

${ }^{8}$ T. Monteiro, C. Boemare, M. J. Soarez, E. Alves, C. Marques, C. McHargue, L. C. Ononye, and L. F. Allard, Nucl. Instrum. Methods Phys. Res. B 191, 638 (2002)

${ }^{9}$ I. T. Yoon, T. W. Kang, M. C. Jeong, M. H. Ham, and J. M. Myoung, Appl. Phys. Lett. 85, 4878 (2004).

${ }^{10}$ Yoon Shon, Young Hae Kwon, Sh. U. Yuldashev, J. H. Leem, C. S. Park, D. J. Fu, H. J. Kim, T. W. Kang, and X. J. Fang, Appl. Phys. Lett. 81, 1845 (2002).

${ }^{11}$ M. A. Reshchikov, H. Morkoç, S. S. Park, and K. Y. Lee, Appl. Phys. Lett. 81, 4970 (2002).

${ }^{12}$ J. Q. Wang, P. P. Chen, H. B. Mao, Z. Q. Zhu, and W. Lu, Physica B 358, 185 (2005).

${ }^{13}$ H. C. Yang, T. Y. Lin, and Y. F. Yen, Phys. Rev. B 62, 12593 (2000).

${ }^{14}$ C. R. Miskys, M. K. Kelly, O. Ambacher, G. Martínez-Criado, and M. Stutzmann, Appl. Phys. Lett. 77, 1858 (2000).

${ }^{15}$ S. Emura, T. Moriga, J. Takizawa, M. Nomura, K. R. Bauchspiess, T. Murata, K. Harada, and H. Maeda, Phys. Rev. B 47, 6918 (1993).

${ }^{16}$ C. Gauthier, H. Emerich, and J. Goulon, Jpn. J. Appl. Phys., Part 132 , 226 (1992).

AIP license or copyright; see http://apl.aip.org/apl/copyright.jsp 